\section{Duração e fatores associados ao aleitamento materno em crianças menores de 24 meses de idade no estado de Pernambuco}

\author{
Duration and associated factors to \\ breastfeeding among children under 24 \\ months in the state of Pernambuco
}

Maria Gorete Lucena de Vasconcelos 1 Pedro Israel Cabral de Lira 2 Marília de Carvalho Lima 3
1 Departamento de Enfermagem. Universidade Federal de Pernambuco. Hospital das Clínicas. Bloco A. Rua Prof. Moraes Rego, s. n. Cidade Universitária. Recife, PE, Brasil. CEP: 50.670901. E-mail: mariagorete47@hotmail.com.br 2 Departamento de Nutrição. Centro de Ciências da Saúde. Universidade Federal de Pernambuco, Recife, PE, Brasil 3 Departamento Materno-Infantil. Universidade Federal de Pernambuco. Hospital das Clínicas. Recife, PE, Brasil

\begin{abstract}
Objectives: to assess breastfeeding time in children under 24 months old and determine factors associated to exclusive breastfeeding in the State of Pernambuco.

Methods : cross sectional study of 852 children aged 24 months old and younger within a sample of 2078 children under five years old selected for the II State Health and Nutrition Survey. Data was collected for the period between February and May, 1997. Survival analysis for median calculation of breastfeeding and housing, per capita income, maternal education level, prenatal care, breastfeeding orientation, delivery type and birthweight was performed.

Results: survival analysis determined exclusive, predominant and total breastfeeding median time of 24.77 and 112 days respectively. Median exclusive breastfeeding time was significantly longer among the children living in the Metropolitan Region of Recife (148 days) whose mothers had incomes above two minimum wages (201 days), who had six or more prenatal medical visits (129 days) and received breastfeeding instructions during prenatal care 126 days). Birthweight, maternal literacy and type of delivery did not significantly influence exclusive breastfeeding.

Conclusions: breastfeeding time in Pernambuco was determined specially in the Metropolitan Region of Recife indicating that prenatal care is one of the opportunity factors to orient and encourage mothers to breastfeed their infants.
\end{abstract}

Key words Breastfeeding, Associated factors, Maternal breastfeeding

\section{Resumo}

Objetivos: avaliar a duração do aleitamento materno em crianças menores de 24 meses de idade no estado de Pernambuco e verificar a associação de fatores com o aleitamento materno total.

Métodos: corte transversal com 852 crianças até 24 meses de idade que fizeram parte da amostra de 2078 menores de cinco anos selecionada para a II Pesquisa Estadual de Saúde e Nutrição. Os dados foram coletados no período de fevereiro a maio de 1997. Utilizou-se a análise de sobrevivência para cálculo da mediana do aleitamento materno e das associações com a situação do domicílio, renda per capita, escolaridade materna, consultas no pré-natal, orientação sobre aleitamento materno, tipo de parto e peso ao nascer.

Resultados: através de análise de sobrevivência verificou-se que a duração mediana do aleitamento materno exclusivo, predominante e total foi de 24, 77 e 112 dias, respectivamente. A duração mediana do aleitamento materno total foi significantemente mais prolongada entre as crianças que residiam na Região Metropolitana do Recife (148 dias), cujas mães percebiam um rendimento familiar per capita acima de dois salários mínimos (201 dias), que haviam freqüentado seis ou mais consultas no pré-natal (129 dias) e recebido orientação sobre aleitamento materno durante o pré-natal (126 dias). O peso ao nascer, a alfabetização materna e o tipo de parto não influenciaram significativamente o aleitamento total.

Conclusões: verificou-se um crescimento na duração do aleitamento materno em Pernambuco, particularmente na Região Metropolitana do Recife, demonstrando ser o pré-natal um dos fatores de oportunidade para orientar e incentivar às mães a amamentarem seus filhos.

Palavras-chave Amamentação, Fatores associados, Aleitamento materno 


\section{Introdução}

A amamentação é a forma de nutrição que mais efetivamente contribui para o crescimento e desenvolvimento da criança devido às suas vantagens de ordem nutricional, imunológicas, econômicas, psicológicas e ecológicas. ${ }^{1,2}$ Após a gestação, a amamentação é a principal alternativa nutricional para a criança, que associa elementos fundamentais da nutrição correta: alimento, saúde e cuidados.

Segundo a Organização Mundial de Saúde (OMS), por ser um alimento completo, o leite humano deve ser fornecido exclusivamente desde o nascimento até os primeiros quatro a seis meses de vida e sua continuidade com alimentos complementares é recomendada até dois anos ou mais. 3 Para a Academia Americana de Pediatria a alimentação ao seio materno é recomendada para todos os recémnascidos a termo e pré-termo vigorosos, por ser nutricionalmente equilibrada e possibilitar um estreito relacionamento mãe-filho. Deve começar tão cedo quanto possível e ser oferecida à livre demanda, sem necessidade de introdução de água, suco ou outros alimentos. 4

No Estatuto da Criança e do Adolescente, 5 artigo nove, o aleitamento materno é tratado como uma questão de direito à vida e à saúde, e como instrumento de direito humano universalmente aceito foi ratificado 191 vezes, segundo o Fundo das Nações Unidas para infância (UNICEF). 6

Estudos convergem opiniões de que o leite materno atua prevenindo a desnutrição e as doenças infecciosas, principalmente as diarréias e infecções respiratórias, importantes causas de morbi-mortalidade infantil.7-13 Outros benefícios relacionados a diabetes, doenças atópicas, cáries, hipovitaminose A e HIV, acham-se relatados na literatura, enfatizando a extensão de alguns desses benefícios, inclusive, até a adolescência. 13-17

Vale salientar que, diante da proteção fornecida pelo aleitamento materno, a mortalidade infantil em famílias de baixo nível socioeconômico, está relacionada principalmente às doenças infecciosas, sendo que nos primeiros seis meses de vida a proteção contra diarréia, apresenta-se substancialmente maior quando comparada as mortes devido às infecções respiratórias agudas. ${ }^{13}$

A prevalência do aleitamento materno varia entre países, regiões e populações urbanas e rurais. Em Toronto, $83 \%$ das mães amamentaram seus filhos após o nascimento e até o quinto mês de vida, $35 \%$ dos lactentes mamavam exclusivamente. 18 Entre crianças chilenas, a prevalência de aleitamento exclusivo aos três meses de idade era de 59\%, encontrando-se uma freqüência do aleitamento materno parcial de $31 \%$ aos doze meses. ${ }^{19} \mathrm{Na}$ América Latina, do nascimento até os dois meses de idade, a freqüência da amamentação variou de $77 \%$ no México a $94 \%$ na Colômbia e Guatemala. ${ }^{20}$

No Brasil, conforme trabalho de Venâncio e Monteiro, 21 a trajetória do aleitamento materno nas décadas de 70 e 80, utilizando dados do Estudo Nacional da Despesa Familiar (ENDEF) e da Pesquisa Nacional de Saúde e Nutrição (PNSN), confirmou um aumento na freqüência e na duração da amamentação, cuja duração mediana da amamentação passou de 2,5 meses para 5,5 meses, entre 1975 e 1989. A PNSN encontrou uma duração do aleitamento materno predominante no país de 72 dias, sendo de 74 dias na zona urbana e 64 dias na zona rural. A maior duração ocorreu na região Centro-Oeste (92 dias) e a menor na região Nordeste (41 dias), enquanto as regiões Sul, Sudeste e Norte apresentaram medianas de 86,82 e 51 dias, respectivamente. Com relação ao aleitamento misto, o Brasil apresentou uma duração mediana de 134 dias, sendo 123 dias para a zona urbana e 191 dias para a zona rural.22 Entretanto, essa duração permaneceu inferior à aquela observada em outros países latinoamericanos, mesmo considerando as possíveis diferenças metodológicas entre os estudos. 20

Na década de 90, a Pesquisa Nacional sobre Demografia e Saúde (PNDS)23 revelou ser bastante alto o percentual de crianças que estavam sendo amamentadas e recebendo complementação, alcançando $41,9 \%$ das crianças de zero a três meses e $45,3 \%$ daquelas entre quatro e seis meses.

Em Pernambuco, a I Pesquisa Estadual de Saúde e Nutrição, realizada em 1991, mostrou um discreto aumento na duração do aleitamento materno total nas áreas rurais em relação à capital, apesar de apresentar menores índices em relação a outros estados do nordeste, como o Maranhão e Piauí, que apresentaram mediana de nove meses e quase sete meses, respectivamente, sendo essa duração em Pernambuco inferior a três meses. 24

Compreende-se que, apesar do fato da duração do aleitamento materno ter se ampliado no país como um todo, a adoção da amamentação resulta da complexa interação de múltiplos fatores, que podem interferir com a freqüência e a duração dessa prática. Portanto, sob essa ótica, a proposta deste estudo é pesquisar a duração do aleitamento materno no estado de Pernambuco e verificar a associação de fatores com o aleitamento materno total. 


\section{Métodos}

Tratou-se de um estudo de corte transversal realizado em 1997, no estado de Pernambuco. A população do estudo foi constituída pelas 852 crianças com até 24 meses de idade que fizeram parte da amostra de 2078 menores de cinco anos, selecionadas para a II Pesquisa Estadual de Saúde e Nutrição. 25 Essa pesquisa considerou três estratos geo-econômicos no estado, Região Metropolitana do Recife (RMR), Interior Urbano (IU) e Interior Rural (IR); sendo a amostra do tipo probabilística (aleatória estratificada) representatividade dos estratos, estabelecendo-se como critério de exclusão para o estudo, as crianças serem filhas de mães adotivas.

O instrumento para coleta de dados foi um questionário pré-codificado sobre as condições socioeconômicas, demográficas, de assistência ao prénatal, duração do aleitamento materno e causas do desmame, aplicado por uma equipe de 15 profissionais de nível superior (psicólogos, sociólogos, assistentes sociais, enfermeiras e nutricionistas), cinco alunas do Mestrado de Nutrição em Saúde Pública da Universidade Federal de Pernambuco (UFPE) e equipes do Programa de Agentes Comunitários de Saúde (PACS) dos municípios.

A definição do tipo de aleitamento materno baseou-se em Labbok et al.26 que consideram: aleitamento exclusivo, quando a criança só recebe leite materno, sem introdução de nenhum outro alimento líquido ou sólido (com exceção de gotas ou xarope de vitaminas, remédios ou sais minerais); aleitamento predominante, quando a principal fonte de alimento da criança é o leite materno, introduzindo líquidos, como água, chá e/ou sucos; e aleitamento total, quando se introduz leite artificial e outros líquidos.

Os dados foram digitados em dupla entrada e processados em microcomputador utilizando o software Epi-info, versão 6,04. Posteriormente, utilizouse o Statistical Package for the Social Science (SPSS), versão 8,0, para a análise de sobrevivência.

A técnica de análise de sobrevivência foi utilizada para cálculo da duração mediana para as três diferentes práticas do aleitamento materno, considerando pelo menos um dia de aleitamento materno para todas as crianças do estudo. 27

O método estatístico para avaliar a associação entre as variáveis explanatórias estudadas (situação do domicílio, renda per capita, escolaridade materna, consultas no pré-natal, orientação sobre aleitamento materno no pré-natal, tipo de parto e peso ao nascer) e o aleitamento materno total foi o teste de Wilcoxon, considerando $p<0,05$ como estatisticamente significante.

Os objetivos da pesquisa e a confidencialidade dos dados foram previamente explicados às famílias, realizando-se a entrevista após a concordância das mesmas, assinando o termo de consentimento livre e esclarecido.

\section{Resultados}

Ao se comparar às curvas de sobrevida das diferentes práticas do aleitamento materno (Figura 1), verificou-se o declínio dos segmentos iniciais das curvas, demonstrando alta taxa de desmame já nos primeiros dias de vida, sendo maior em relação ao aleitamento exclusivo. A duração mediana do aleitamento exclusivo, predominante e total foi respectivamente, 24,77 e 112 dias.

A Tabela 1 apresenta a associação entre a duração do aleitamento materno total e as variáveis explanatórias estudadas. $\mathrm{Na}$ amostra, $36,1 \%$ dos domicílios localizavam-se na Região Metropolitana do Recife, enquanto $63,9 \%$ estavam distribuídos no Interior Urbano (32,2\%) e Rural (31,7\%). Considerando-se a renda, 79,4\% das famílias recebiam menos de um salário mínimo per capita. Com relação à escolaridade materna, 28,9\% das mães eram analfabetas. Quanto ao pré-natal 16,6\% não o realizaram. Entre as crianças, o baixo peso ao nascer representou $7,4 \%$ da amostra considerada.

Verificou-se que a duração do aleitamento materno total foi significantemente maior entre as

Figura 1

Práticas do aleitamento materno. Pernambuco, 1997.

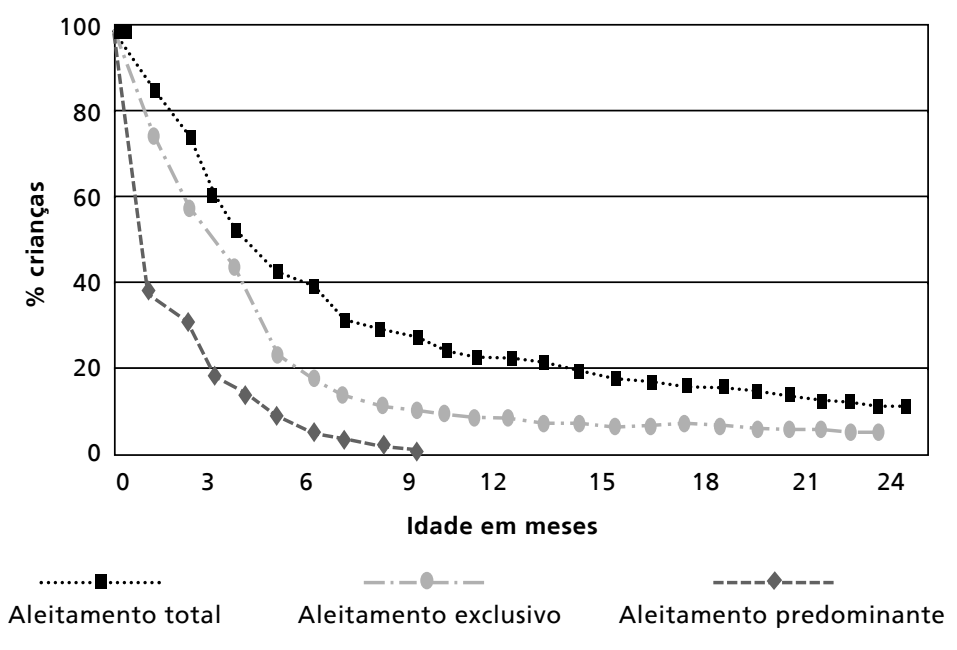


Duração do aleitamento materno total em menores de 24 meses de idade no estado de Pernambuco segundo algumas variáveis explanatórias estudadas.

\begin{tabular}{|c|c|c|c|c|}
\hline Variável & $\mathrm{N}=852$ & $\%$ & Mediana (dias) & Teste estatístico \\
\hline \multicolumn{5}{|l|}{ Situação do domicílio } \\
\hline Região Metropolitana do Recife & 308 & 36,1 & 148 & $* \mathrm{~W}=27,03$ \\
\hline Interior Urbano & 274 & 32,2 & 90 & $p<0,001$ \\
\hline Interior Rural & 270 & 31,7 & 110 & \\
\hline \multicolumn{5}{|l|}{ Renda per capita (salário mínimo)** } \\
\hline$<1$ & 671 & 79,4 & 108 & $W=7,90$ \\
\hline $1-2$ & 90 & 10,6 & 108 & $p=0,02$ \\
\hline$>2$ & 84 & 10,0 & 201 & \\
\hline \multicolumn{5}{|l|}{ Escolaridade materna } \\
\hline Alfabetizada & 606 & 71,1 & 117 & $W=2,67$ \\
\hline Analfabeta & 246 & 28,9 & 105 & $p=0,10$ \\
\hline \multicolumn{5}{|l|}{ Consultas no pré-natal*** } \\
\hline Não & 140 & 16,6 & 91 & $W=14,54$ \\
\hline $1-5$ & 319 & 37,6 & 115 & $p<0,001$ \\
\hline$\geq 6$ & 388 & 45,8 & 129 & \\
\hline \multicolumn{5}{|c|}{ Orientação sobre aleitamento no pré-natal ${ }^{* * * *}$} \\
\hline Sim & 589 & 83,4 & 126 & $W=4,04$ \\
\hline Não & 117 & 16,6 & 101 & $p=0,04$ \\
\hline \multicolumn{5}{|l|}{ Tipo de parto } \\
\hline Normal & 609 & 71,5 & 110 & $W=0,00$ \\
\hline Cesariana & 243 & 28,5 & 121 & $p=0,99$ \\
\hline \multicolumn{5}{|l|}{ Peso ao nascer (gramas)***** } \\
\hline$<2500$ & 60 & 7,4 & 123 & $W=0,63$ \\
\hline $2500-2999$ & 164 & 20,1 & 107 & $p=0,7$ \\
\hline$>2999$ & 588 & 72,5 & 112 & \\
\hline
\end{tabular}

*W = Teste de Wilcoxon; ** 7 casos sem informação; *** 5 casos sem informação; **** Entre 706 mães que realizaram pré-natal; ***** 40 casos sem informação.

Fonte: INAM. II Pesquisa Estadual de Saúde e Nutrição. Recife; 1997.25

mães residentes na RMR (148 dias) em relação aquelas do IR (110 dias) e do IU (90 dias) $(p<0,001)$.

Entre as mães com renda familiar per capita acima de dois salários mínimos a prevalência da amamentação foi maior (201 dias) do que nos dois grupos inferiores de renda (108 dias), com diferença significante $(p=0,02)$.

As mães que freqüentaram seis ou mais consultas no pré-natal apresentaram duração mediana do aleitamento materno maior (129 dias) que as mães com um número inferior a seis consultas (115 dias) e aquelas que não fizeram o pré-natal (91 dias), com uma associação estatisticamente significante $(p<0,001)$.

As orientações fornecidas às mães sobre aleitamento recebidas no pré-natal também contribuíram significativamente para o aumento da mediana do aleitamento total (126 dias), em relação às que não receberam esse tipo de orientação (101 dias) com valores de $p=0,04$.

Com relação às demais variáveis explanatórias estudadas (escolaridade materna, tipo de parto e peso ao nascer) não houve associação estatisticamente com o aleitamento materno total (Tabela 1).

\section{Discussão}

No presente estudo, a duração mediana do aleitamento materno foi realizada através da técnica de análise de sobrevivência, cujas informações sobre a prática da amamentação mereceram atenção na interpretação, frente a possíveis viés de memória das mães entrevistadas. 
Desse modo, quando se comparou a situação do aleitamento materno nesse estudo, com os dados da Pesquisa Estadual de Saúde e Nutrição 28 verificouse que houve crescimento na duração mediana, elevando-se de 89 dias para 112 dias na presente pesquisa. Com relação ao aleitamento materno exclusivo, uma pesquisa realizada nas capitais brasileiras em 2001,29 encontrou uma mediana deste tipo de aleitamento de 23,4 dias, semelhante à encontrada neste estudo.

Observou-se, ainda, que no estado houve uma inversão no panorama da amamentação, pelo aumento que ocorreu na duração mediana na Região Metropolitana (148 dias) em relação ao interior. Possivelmente, o acesso facilitado aos serviços de saúde nas regiões urbanas e as estratégias adotadas pelas políticas públicas de incentivo e promoção do aleitamento materno, poderiam responder pela mudança no quadro encontrado. Nesse sentido, acredita-se também que o esforço dos profissionais de saúde envolvidos com o ensino e a pesquisa sobre a assistência materno-infantil, promovendo e apoiando o aleitamento materno, tenha contribuído para esta mudança. Em contra-posição, Passos, 30 em seus estudos realizados em Ouro Preto, Minas Gerais, não encontrou associação em relação à duração do aleitamento materno e o fato de as mulheres residirem em áreas urbanas ou rurais. Porém, na área urbana de São José do Rio Preto, em São Paulo, uma avaliação das práticas de alimentação infantil mostrou uma duração mediana da amamentação de 205 dias. 31

Assim, acredita-se que a proximidade com um maior número de serviços de saúde qualificados, o acesso facilitado a esses e às informações sobre os benefícios da amamentação, inclusive pelos meios de comunicação, contribuem para o estímulo e promoção do aleitamento materno.

A renda familiar apresentou-se como fator associado à duração do aleitamento materno total, contribuindo para o aumento da mediana entre aquelas mães cujas famílias apresentaram rendimento maior do que dois salários mínimos per capita. Talvez a melhor condição de renda tenha influído sobre o nível educacional das mães facilitando, conseqüentemente, o acesso às informações sobre a importância da amamentação. De outro modo, entre as mães com renda inferior, que necessitavam contribuir com seu trabalho para o sustento da família, a amamentação poderia ter sido prejudicada pela falta de acesso a essas informações. O problema poderia intensificar-se, principalmente, em relação àquelas que trabalhavam sem o amparo legal da legislação trabalhista. Evidências dessa natureza já foram levantadas em outros estudos.32,33 Além do trabalho informal, o desemprego também pode interferir na prática do aleitamento materno, sendo considerado um fator de risco para o desmame, como demonstrou o estudo realizado em 84 municípios do estado de São Paulo. 34

A decisão de amamentar raramente é feita antes do nascimento da criança, fato que confere ao prénatal ótima oportunidade para orientar e incentivar às mães a amamentarem seus filhos. O estudo mostrou, também, que ocorreu uma relação diretamente proporcional entre número de consultas realizadas no pré-natal e o aumento da duração mediana do aleitamento total. Adair et al.35 e Barnes et al.36 realizaram investigações que corroboram os achados.

Entretanto, não se deve considerar apenas o espaço (pré-natal) em que a ação se desenvolve (decisão de amamentar). O profissional de saúde deve colocar-se na perspectiva do sujeito (mãe) que vivencia a amamentação (fenômeno histórico e social) em um contexto temporal dinâmico.

Javorski37 afirma que muitas vezes os instrumentos de trabalho utilizados são impositivos, autoritários e impessoais e não têm contribuído com a decisão da mãe de amamentar, existindo etapas na estrutura emocional familiar que podem interferir nessa decisão. Seguindo a mesma linha de pensamento, Silva38 enfatiza que determinação de amamentar, sua qualidade e duração dependem do significado que a mulher atribui a essa experiência, através de elementos de interação vivenciados por ela em seu contexto. As ações de incentivo ao aleitamento materno desenvolvidas pelo profissional de saúde devem estar embasadas na noção de direitos reprodutivos, direcionando-as na perspectiva de resgatar a visibilidade da mulher enquanto sujeito principal da prática da amamentação. ${ }^{39}$ Rotenberg e De Vargas 40 percebem como única a experiência da amamentação em relação a cada filho e considerando-a como um processo, compreendem, na dimensão temporal, dois momentos críticos: o início e o término, ou seja, o estabelecimento da amamentação propriamente dita e o desmame total.

Nessa perspectiva, Vasconcelos 41 relata que, acompanhando no domicílio crianças nascidas antes do termo e com baixo peso ao nascer, egressas de uma instituição participante da "Iniciativa Hospital Amigo da Criança", encontrou algumas desmamadas antes do terceiro mês de vida, apesar das inúmeras intervenções de incentivo ao aleitamento materno exclusivo, fornecidas à mãe durante o período de permanência com o filho hospitalizado. Assim, entende-se a necessidade das mães serem continua- 
mente apoiadas após a alta hospitalar pelos profissionais que atuam nos programas de saúde da família e agentes comunitários de saúde, sendo esse apoio, mais recentemente, reforçado por outros pesquisadores. 42

Alguns autores constataram que a ausência do programa "Hospital Amigo da Criança", foi um fator determinante de risco para o desmame 34,43 e observam que o tempo de amamentação, aumenta expressivamente com a adoção dessa estratégia, 44 recomendando a sua implementação, como também, a de Bancos de Leite Humano. 31

É possível que o desmame acentuado verificado no estado de Pernambuco logo nos primeiros meses de vida da criança venha a ser reduzido com intervenções e estratégias dessa natureza, sem esquecer de compreender e buscar contribuições em outras formas de abordagem sobre a questão da amamentação. Assim, pesquisando, monitorando e orientando as ações de incentivo à amamentação no prénatal e no domicílio através do programa saúde da família e de agentes comunitários de saúde, poderemos dar um salto qualitativo sobre sua duração.

Em suma, os resultados apontaram para um crescimento na duração do aleitamento materno em Pernambuco, particularmente na Região Metropolitana do Recife, demonstrando ser o pré-natal um

\section{Referências}

1. Serva VB. Manejo da lactação. In: Lima GS, Braga TDA, Meneses JA, editores. Neonatologia (IMIP). Rio de Janeiro: Medsi; 2004. p. 75-94

2. Coutinho SB. Aleitamento materno. In: Silva AS, editor. Manual de neonatologia. Rio de Janeiro: Medsi; 2002. p. 122

3. Rea MF. Amamentação: a visão das mulheres e a Semana Mundial [Editorial]. J Pediatr [Rio J] 1995; 71: 179.

4. Rea MF. A amamentação e o uso do leite humano: o que recomenda a Academia Americana de Pediatria. J Pediatr [Rio J] 1998; 74: 171-3.

5. Ministério da Saúde. Estatuto da criança e do adolescente. Brasília (DF), 1991.

6. UNICEF (Fundo das Nações Unidas para Infância). Situação mundial da infância. Brasília (DF): 1998.

7. Sazawal S, Bhan MK, Bhandari N. Typy of milk feeding during acute diarrhoea and the risk of persistent diarrhoea: a case control study. Acta Paediatr 1992; 381 (Suppl): 93-7.

8. Victora CG, Fuchs SC, Flores AJC. Risk factors for pneumonia among children in a Brazilian metropolitan area.. Pediatrics 1994; 93: 977-85.

9. Chaves SP, Lei DLM, Lerner BR. Aleitamento, estado nutricional e morbidade no primeiro ano de vida. Rev Nutr 1995; 8: 31-46. dos fatores de oportunidade para orientar e incentivar as mães a amamentarem seus filhos.

De outro modo, este estudo poderá ampliar e atualizar os diagnósticos situacionais de saúde e nutrição do grupo materno-infantil no Estado, fornecendo subsídios às políticas e programas governamentais, especificamente em relação à duração e aos determinantes do aleitamento materno em crianças com até 24 meses de idade.

Recomenda-se, ainda, a construção de outras abordagens metodológicas a serem utilizadas sobre o tema estudado, buscando elucidar fatos que possam melhorar as ações desenvolvidas no ambien-te hospitalar e no domicílio.

\section{Agradecimentos}

Às famílias participantes, a equipe de entrevistadores do trabalho de campo e aos coordenadores da II Pesquisa Estadual de Saúde e Nutrição, financiada pelo Ministério da Saúde. Ao CNPq pelas Bolsas de Produtividade em Pesquisa dos Prof. Pedro Lira e Marilia Lima.

10. Costa FS, Gomes VC, Martines J. Case-control study of risk of dehydrating diarrhoea in infants in vulnerable period after full weaning. Br Med J 1996; 313: 391-4.

11. Escuder MM, Venancio SI, Pereira JC. Impact estimates of breastfeeding over infant mortality. Rev Saude Publica 2003; 37: 319-25.

12. Saleemi MA, Zaman S, Akhtar HZ, Jalil F, Ashraf RN, Hanson LA, Mellander L. Feeding patterns, diarrhoeal illness and linear growth in 0-24 month-old children. J Trop Pediatr 2004; 50: 164-9.

13. Victora CG. Effect of breastfeeding on infant and child mortality due to infectious disease in less developed countries: a pooled analysis. Lancet 2000; 355: 451-5.

14. Virtanem SM, Rasanen L, Ylonen K. Early introducion of dairy products associated with increased risk of IDDM in Finnish children. Diabetes 1993; 42: 1786-90.

15. Saarinen UM, Kajosaari M. Breastfeeding as prophylaxis atopic disease: a prospective follow-up study until 17 years old. Lancet 1995; 346: 1065-9.

16. Al-Dashti AA, Williams SA, Curzon MEJ. Breastfeeding, bottle feeding and dental caries in Kuwait, a country with low-fluoride levels in the water supply. Comm Dental Health $1995 ; 12: 42-7$.

17. Khatry SK, West KP, Katz J. Epidemiology of xerophthalmia in Nepal. Arch Ophthalmol 1995; 113: 425-9. 
18. Barber CM, Abernathy T, Steinmetz B. Using a breastfeeding prevalence survey to identify a population for target programs. Can J Public Health 1997; 88: 242-5.

19. Castilho C, Atalah E, Riumallo C. Breastfeeding and the nutritional status of nursing children in Chile. Bull Pan-Am Health Organ 1996; 30: 125-33.

20. Pérez-Escamilla R. Patrones de la lactancia natural en América Latina y el Caribe. Bol Ofic Sanit Panam 1993; 115: 185-94.

21. Venancio SI, Monteiro CA. Tendência da prática da amamentação no Brasil nas décadas de 70 e 80. Rev Bras Epidemiol 1998; 1: 40-9.

22. INAN (Instituto Nacional de Alimentação e Nutrição). Pesquisa Nacional sobre Saúde e Nutrição (1989): resultados preliminares. Brasília (DF); 1990.

23. BEMFAN (Sociedade Civil do Bem-Estar Familiar no Brasil). Pesquisa Nacional sobre Demografia e Saúde. Programa de Pesquisas de Demografia e Saúde. Rio de Janeiro; 1996.

24. UNICEF (Fundo das Nações Unidas para a Infância). Saúde e nutrição das crianças nordestinas: pesquisas estaduais 1987 - 1992. Brasília (DF); 1995.

25. INAN (Intituto Nacional de Alimentação e Nutrição). II Pesquisa Estadual de Saúde e Nutrição. Recife; 1998.

26. Labbok M, Krasovec K. Towards consistency in breastfeeding definitions. Stud Fam Plan 1990; 21: 226-30.

27. Barros FC, Victora CG. Epidemiologia da saúde infantil: um manual para diagnósticos comunitários. São Paulo: Hucitec; 1991.

28. Pernambuco. Governo. Pesquisa Estadual de Saúde e Nutrição. Recife; 1992.

29. Ministério da Saúde. Secretaria de Políticas de Saúde. Área de Saúde da Criança. Pesquisa de prevalência do aleitamento materno nas capitais e no Distrito Federal. Brasília (DF); 2001.

30. Passos MC. Epidemiologia do desmame precoce em crianças de 0 a 24 meses no município de Ouro Preto [dissertação mestrado]. Recife: Departamento de Nutrição, Centro de Ciências da Saúde da Universidade Federal de Pernambuco; 1997.

31. Figueiredo MG, Sartorelli DS, Zan TAB, Garcia E, Silva LC, Carvalho FLP, Pascotto RC, Macri S, Cardoso MA. Inquérito de avaliação rápida das práticas de alimentação infantil em São José do Rio Preto, São Paulo, Brasil. Cad Saúde Pública 2004; 20: 172-9.
32. Lockin MP. Telling the world: low-income women and their breastfeeding experiences. J Hum Lact 1995; 11: 285-91.

33. Rea MF, Venancio SI, Batista LE. Possibilidades e limitações da amamentação entre mulheres trabalhadoras formais. Rev Saúde Pública 1997; 31: 149-56.

34. Venancio SI, Escuder MML, Kitoko P, Rea MF, Monteiro CA. Freqüência e determinantes do aleitamento materno em municípios do estado de São Paulo. Rev Saúde Pública 2002; 36: 313-8.

35. Adair LS, Popkin BM, Guilkey DK. The duration of breastfeeding: how is it affected by biological, sociodemographic, health sector and food industry factors? Demography 1993; 30: 63-80.

36. Barnes J, Stein A, Smith T. Extreme attitudes to body shape, social and psychological factors and a reluctance to breast feed. J R Soc Med 1997; 90: 551-9.

37. Javorski M. Os significados do aleitamento materno para as mães de prematuros em cuidado Canguru [dissertação mestrado]. Ribeirão Preto: Escola de Enfermagem de Ribeirão Preto da Universidade de São Paulo; 1997.

38. Silva IA. Desvendando as faces da amamentação através da pesquisa qualitativa. Rev Bras Enferm 2000; 53: 241-9.

39. Moreira KFA, Nakano MAS. Aleitamento materno: instintivo? natural? O paradigma biológico x os direitos reprodutivos em discussão. Rev Bras Enferm 2002; 55: 685-90.

40. Rotenberg S, De Vargas S. Práticas alimentares e o cuidado da saúde: da alimentação da criança à alimentação da família. Rev Bras Saúde Matern Infant 2004; 4: 71-83.

41. Vasconcelos MGL. Implantação de um grupo de apoio à mãe acompanhante de recém-nascido pré-termo e de baixo peso em um hospital amigo da criança na cidade de Recife, PE [tese doutorado]. Ribeirão Preto: Escola de Enfermagem de Ribeirão Preto da Universidade de São Paulo; 2004.

42. Marques RFSV, Lopez FA, Braga JAP. O crescimento de crianças alimentadas com leite materno exclusivo nos primeiros seis meses de vida. J Pediatr [Rio J] 2004; 80: 99105.

43. Audi CAF, Corrêa MAS, Latorre MRDO. Alimentos complementares e fatores associados ao aleitamento materno e ao aleitamento materno exclusivo em lactentes até 12 meses de vida em Itapira, São Paulo, 1999. Rev Bras Saúde Matern Infant 2003; 3: 85-93.

44. Escuder MML, Venâncio SI, Pereira JCR. Estimativa de impacto da amamentação sobre a mortalidade infantil. Rev Saúde Pública 2003; 37: 319-25.

Recebido em 29 de setembro de 2004

Versão final apresentada em 14 de setembro de 2005

Aprovado em 28 de setembro de 2005 